\title{
Sandwich results for periodicity and conjugacy
}

\author{
Janusz Matkowski and Pawę WóJCik@D
}

\begin{abstract}
Let $P$ be a nonconstant selfmap of a set $\mathcal{M}$. A sandwich-type theorem for generalized sub- $P$-periodic functions defined on $\mathcal{M}$ with values in a reflexive Banach space is proved. In particular, given functions $f, g: \mathcal{M} \rightarrow \mathbb{R}$, we obtain necessary and sufficient conditions for the existence of a generalized $P$-periodic function $F: \mathcal{M} \rightarrow \mathbb{R}$ such that $f \leq F \leq g$. The formula for $F$ is given and its Lipschitz constant is discussed. Moreover the solvability of the functional equation $f \circ p=r \circ f$ with the help of a new sandwich method, is considered.
\end{abstract}

Mathematics Subject Classification. Primary 39B82, 39B12; Secondary 39B62, 46B20.

Keywords. Sandwish theorem, Functional equation, Functional inequality, Periodicity, Conjugacy, Invariant mean, Schauder principle.

\section{Introduction}

In a recent paper Izumi et al. [5] proved the following sandwich (separation) type result: functions $f, g: \mathbb{R} \rightarrow \mathbb{R}$ satisfy the inequalities

$$
f(x+n p) \leq g(x), \quad n \in \mathbb{Z}, x \in \mathbb{R},
$$

for some $p \in \mathbb{R} \backslash\{0\}$ if, and only if, there is a p-periodic function $F: \mathbb{R} \rightarrow \mathbb{R}$ separating $f$ and $g$, that is such that $F(x+p)=F(x)$ for all $x \in \mathbb{R}$ and $f \leq F \leq g$.

In Sect. 2 of this paper, applying Gajda's theorem on the existence of a translative invariant mean in the Banach space of bounded vector valued functions [4], we prove far reaching generalizations of this result. Let $\mathcal{M}$ be a nonempty set, $P: \mathcal{M} \rightarrow \mathcal{M}$ a nonconstant mapping, and $\mathfrak{X}$ a reflexive Banach space. Assuming that a function $f: \mathcal{M} \rightarrow \mathfrak{X}$ and a set-valued function $\Gamma: \mathcal{M} \rightarrow 2^{\mathfrak{X}}$ with bounded closed and convex values are such that

The research of Paweł Wójcik and this paper were partially supported by National Science Centre, Poland under Grant Miniatura 2, No. 2018/02/X/ST1/00313. 


$$
f\left(P^{n}(x)\right) \in \Gamma(x), \quad n \in \mathbb{N}_{0}, x \in \mathbb{R}
$$

(where $\mathbb{N}_{0}:=\{0,1, \ldots$,$\} ), we prove that there is a P$-periodic selection $F$ of $\Gamma$ (i.e. such that $F \circ P=F$ ) (Theorem 2). Assume that $h: \mathcal{M} \rightarrow \mathbb{R}$ is $P$-super-periodic (i.e. $h \circ P \geq h$ ) and $g: \mathcal{M} \rightarrow \mathbb{R}$ is $P$-sub-periodic (i.e. $h \circ P \leq h)$. Applying Theorem 2 with $\mathfrak{X}=\mathbb{R}$ we prove the inequality $h \leq g$ holds if and only if there is a $P$-periodic function separating $h$ and $g$ (Theorem 3). A strengthening of Theorem 3 is given. At the end of this section, assuming that $\mathcal{M}$ is a metric space, we give conditions under which the Lipschitz continuity of a $(P-\Gamma)$-periodic function $f$ (see Sect. 2), implies the existence of the Lipschitz continuous $P$-periodic function $F$.

In the context of the periodic equation $f \circ P=f$, with the notation $p=P$, it becomes quite natural to consider a more general functional equation $f \circ p=$ $g \circ f$ linked both to conjugacy and commutativity. A description of solutions of this equations, as well as some related topics, can be found in the papers $[2,3,7,8,10]$. They focus on the existence and uniqueness of continuous and homeomorphic solutions in the case when $p$ and $g$ are given self-maps of a real interval or the unit circle $S_{1}$. In [9], Zdun investigated bounded and continuous solutions $f:[0,1] \rightarrow Y$, where $Y$ is a complete metric space, of the system of equations $f \circ p_{k}=g_{k} \circ f, k=0,1, \ldots, n-1$. Ciepliński and Leśniak dealt with these problems in [1]. Moreover, Kopeček [6] investigated the equation $f \circ p=g \circ f$ for given functions $p$ and $g$ defined on subsets of all real numbers. He obtained a nice characterization of solvability of the above equation in the case when $p, g$ are strictly increasing and continuous. Motivated by results of Kopeček, we investigate similar topics in Sect. 3. Namely, we prove sandwich type results for the functional equation $f(p(x))=r(f(x))$. We conclude this paper with results concerning the functional equation

$$
\alpha f(p(x))=g(f(x)), \quad x \in \mathcal{M},
$$

and the functional inequality

$$
\alpha f\left(p^{n}(x)\right) \geq g^{n}(f(x)), \quad x \in \mathcal{M},
$$

where the constant $\alpha$ and the functions $p: \mathcal{M} \rightarrow \mathcal{M}$ and $g: \mathfrak{X} \rightarrow \mathfrak{X}$ are given.

\section{Generalized periodicity}

Let $S$ be a semigroup and $\mathfrak{X}$ be a Banach space. By $\mathcal{B}(S, \mathfrak{X})$ we denote the space of bounded $\mathfrak{X}$-valued functions with the sup-norm $\|\cdot\|_{\infty}$. In our consideration the following result due to Gajda [4, Th.2.1, p. 20] is important.

Theorem 1. [4] Let $(S,+)$ be a commutative semigroup. Suppose that $\mathfrak{X}$ is reflexive. There is a continuous linear operator $M: \mathcal{B}(S, \mathfrak{X}) \rightarrow \mathfrak{X}$ such that

(i) $M\left(\varphi_{a}\right)=M(\varphi)$ for all $a \in S$ and $\varphi \in \mathcal{B}(S, \mathfrak{X})$, where $\varphi_{a}$ denotes the translation of $\varphi$ defined by $\varphi_{a}(x):=\varphi(x+a), x \in S$; 
(ii) for every $\varphi \in \mathcal{B}(S, \mathfrak{X})$ and closed convex bounded subset $V$ of $\mathfrak{X}$, if $\varphi(S) \subseteq$ $V$, then $M(\varphi) \in V$.

The linear operator $M$ is called an invariant mean.

In the sequel $\mathcal{M}$ is a nonempty set, $P: \mathcal{M} \rightarrow \mathcal{M}$ is a nonconstant function, $\mathfrak{X}$ is a reflexive Banach space, and $\Gamma: \mathcal{M} \rightarrow 2^{\mathfrak{X}}$ a set-valued function such that, for every $x$, the value $\Gamma(x) \varsubsetneqq \mathfrak{X}$ is nonempty and convex.

We say that the function $F: \mathcal{M} \rightarrow \mathfrak{X}$ is $P$-periodic [resp. $P$ - $\Gamma$-periodic] if

$$
\forall x \in \mathcal{M} \quad F(P(x))=F(x) \quad[\operatorname{resp} . F(P(x)) \in \Gamma(x)] .
$$

To summarize, the paper [5] and conditions (1), (2) motivate this paper.

Put $\mathbb{N}_{0}:=\mathbb{N} \cup\{0\}$. Now we prove the first main result of this paper.

Theorem 2. Let $\mathcal{M}$ be a nonempty set and let $\mathfrak{X}$ be a reflexive Banach space. Assume that $P: \mathcal{M} \rightarrow \mathcal{M}$ is not a constant mapping. Suppose that $\Gamma: \mathcal{M} \rightarrow 2^{\mathfrak{X}}$ is a set-valued function such that for all $x \in \mathcal{M}$ the set $\Gamma(x)$ is nonempty, bounded, closed and convex. Suppose that a function $f: \mathcal{M} \rightarrow \mathfrak{X}$ satisfies the condition

$$
f\left(P^{n}(x)\right) \in \Gamma(x), \quad x \in \mathcal{M}, n \in \mathbb{N}_{0} .
$$

Then there is a P-periodic function $F: \mathcal{M} \rightarrow \mathfrak{X}$ such that

$$
F(x) \in \bigcap_{m \in \mathbb{N}} \overline{\operatorname{conv}}\left\{f\left(P^{n}(x)\right): n>m\right\}, \quad x \in \mathcal{M} ;
$$

in particular, $F(x) \in \Gamma(x)$ for all $x \in \mathcal{M}$.

Proof. Fix $a \in \mathfrak{X}, \varepsilon \in(0,1), x \in \mathcal{M}$. By the definition of $\operatorname{dist}(a, A)$, there is a $d(x) \in \mathfrak{X}$ such that

$$
\begin{aligned}
& d(x) \in \overline{\operatorname{conv}}\left\{f\left(P^{n}(x)\right): n \in \mathbb{N}_{0}\right\} \text { and } \\
& \|d(x)-a\|-\varepsilon<\operatorname{dist}\left(a, \operatorname{conv}\left\{f\left(P^{n}(x)\right): n \in \mathbb{N}_{0}\right\}\right) .
\end{aligned}
$$

Given $y \in \mathcal{M}$ we define a function $\varphi^{y}: \mathbb{N}_{0} \rightarrow \mathfrak{X}$ by $\varphi^{y}(k):=d\left(P^{k}(y)\right)$. Put $\varphi_{1}^{y}(k):=\varphi^{y}(k+1)$; see (i). It follows that $\varphi^{P(y)}(k)=d\left(P^{k}(P(y))\right)=$ $d\left(P^{k+1}(y)\right)=\varphi^{y}(k+1)=\varphi_{1}^{y}(k)$, which means

$$
\varphi^{P(y)}=\varphi_{1}^{y} \quad \text { for all } y \in \mathcal{M} .
$$

By Theorem 1 (with $S=\mathbb{N}_{0}$ ) there is an invariant mean $M: \mathcal{B}\left(\mathbb{N}_{0}, \mathfrak{X}\right) \rightarrow \mathfrak{X}$. Now we define $F: \mathcal{M} \rightarrow \mathfrak{X}$ by

$$
F(x):=M\left(\varphi^{x}\right) \quad \text { for } \quad x \in \mathcal{M} .
$$

Applying in turn (5) and (i), we have $F(P(x))=M\left(\varphi^{P(x)}\right)=M\left(\varphi_{1}^{x}\right)=$ $M\left(\varphi^{x}\right)=F(x)$, so the function $F$ is $P$-periodic. It is easy to see that

$$
d\left(P^{k}(x)\right) \in \operatorname{conv}\left\{f\left(P^{n+k}(x)\right): n \in \mathbb{N}_{0}\right\} \subseteq \operatorname{conv}\left\{f\left(P^{n}(x)\right): n \in \mathbb{N}_{0}\right\} .
$$


Thus we get $d\left(P^{k}(x)\right) \in \overline{\operatorname{conv}}\left\{f\left(P^{n}(x)\right): n \in \mathbb{N}_{0}\right\} \subseteq \Gamma(x)$ for all $k$ in $\mathbb{N}_{0}$. Therefore, $\varphi^{x}\left(\mathbb{N}_{0}\right) \subseteq \overline{\operatorname{conv}}\left\{f\left(P^{n}(x)\right): n \in \mathbb{N}_{0}\right\} \subseteq \Gamma(x)$, and so by (ii) we obtain $F(x)=M\left(\varphi^{x}\right) \in \overline{\operatorname{conv}}\left\{f\left(P^{n}(x)\right): n \in \mathbb{N}_{0}\right\}$.

Putting $P^{k}(x)$ in place of $x$ in the above equality we get $F\left(P^{k}(x)\right) \in$ $\overline{\operatorname{conv}}\left\{f\left(P^{n}\left(P^{k}(x)\right)\right): n \in \mathbb{N}_{0}\right\}$. On the other hand, we also have $F\left(P^{k}(x)\right)=$ $F(x)$, hence $\left.F(x) \in \overline{\operatorname{conv}}\left\{f\left(P^{n+k}(x)\right)\right): n \in \mathbb{N}_{0}\right\}$. Since $k \in \mathbb{N}_{0}$ is arbitrarily chosen, we conclude that $\left.F(x) \in \bigcap_{m \in \mathbb{N}} \overline{\operatorname{conv}}\left\{f\left(P^{n}(x)\right)\right): n>m\right\}$.

Theorem 3. Let $\mathcal{M}$ be a nonempty set and assume that $P: \mathcal{M} \rightarrow \mathcal{M}$ is not a constant mapping. Suppose that $h: \mathcal{M} \rightarrow \mathbb{R}$ is a $P$-super-periodic function, i.e. $h(P(\cdot)) \geq h(\cdot)$, and $g: \mathcal{M} \rightarrow \mathbb{R}$ is a P-sub-periodic, i.e. $g(P(\cdot)) \leq g(\cdot)$. Then the functions $h, g: \mathcal{M} \rightarrow \mathbb{R}$ satisfy the condition

$$
\forall_{x \in \mathcal{M}} h(x) \leq g(x),
$$

if and only if there is a P-periodic function $F: \mathcal{M} \rightarrow \mathbb{R}$ such that $h \leq F \leq g$.

Proof. Assume (7). Define $f: \mathcal{M} \rightarrow \mathbb{R}$ by $f(x):=\frac{1}{2} h(x)+\frac{1}{2} g(x)$. By the assumptions we have $h\left(P^{n}(x)\right) \geq h(x)$ and $g\left(P^{n}(x)\right) \leq g(x)$. It follows that $h(x) \leq f\left(P^{n}(x)\right) \leq g(x)$ for all $x$ in $M$. For the set-valued function $\Gamma: \mathcal{M} \rightarrow$ $2^{\mathbb{R}}$ given by $\Gamma(x):=[h(x), g(x)]$ we have $f\left(P^{n}(x)\right) \in \Gamma(x)$. Now applying Theorem 2 we arrive at the desired assertion. The converse implication has a trivial verification.

The above result can be strengthened (in some circumstances) as follows.

Theorem 4. Let $\mathcal{M}$ be a nonempty set assume that $P: \mathcal{M} \rightarrow \mathcal{M}$ is not a constant mapping. Suppose that $h: \mathcal{M} \rightarrow \mathbb{R}$ is a P-super-periodic function. Then the functions $h, g: \mathcal{M} \rightarrow \mathbb{R}$ satisfy the condition

$$
\forall_{x \in \mathcal{M}} \forall_{n \in \mathbb{N}_{0}} h\left(P^{n}(x)\right) \leq g(x),
$$

if and only if there is a P-periodic function $F: M \rightarrow \mathbb{R}$ such that $h \leq F \leq g$.

Proof. Assume (8). It is clear that $h(x) \leq h\left(P^{n}(x)\right) \leq g(x)$. Define $\widehat{g}: \mathcal{M} \rightarrow \mathbb{R}$ by $\widehat{g}(x):=\sup \left\{h\left(P^{n}(x)\right): n \in \mathbb{N}_{0}\right\}$. It is easy to check that $h(x) \leq \widehat{g}(x) \leq$ $g(x)$ and $\left\{h\left(P^{n+1}(x)\right): n \in \mathbb{N}_{0}\right\} \subseteq\left\{h\left(P^{n}(x)\right): n \in \mathbb{N}_{0}\right\}$. Therefore we obtain $\widehat{g}(P(x))=\sup \left\{h\left(P^{n+1}(x)\right): n \in \mathbb{N}_{0}\right\} \leq \sup \left\{h\left(P^{n}(x)\right): n \in \mathbb{N}_{0}\right\}=$ $\widehat{g}(x)$, which means that $\widehat{g}$ is $P$-sub-periodic. Moreover $h(x) \leq \widehat{g}(x)$. Applying Theorem 3, we complete the proof. The converse result is immediate.

The next result shows that the Lipschitz property of a $P$ - $\Gamma$-periodic function can be inherited by some $P$-periodic function.

Theorem 5. Let $(\mathcal{M}, \varrho)$ be a metric space and $\mathfrak{X}$ a reflexive Banach space. Suppose that $P: \mathcal{M} \rightarrow \mathcal{M}$ is a uniformly Lipschitz function, i.e. there is $\gamma>0$ such that

$$
\varrho\left(P^{n}(x), P^{n}(y)\right) \leqslant \gamma \cdot \varrho(x, y) \text { for } x, y \in \mathcal{M} \text {; }
$$


$\Gamma: \mathcal{M} \rightarrow 2^{\mathfrak{X}}$ is a set-valued function such that for all $x \in \mathcal{M}$ the set $\Gamma(x)$ is bounded, closed, convex; and $f: \mathcal{M} \rightarrow \mathfrak{X}$ is a P-Г-periodic function. If $f$ is a Lipschitz function with Lipschitz constant $\beta$, then there is a P-periodic function $F: \mathcal{M} \rightarrow \mathfrak{X}$ such that $F$ is a function with Lipschitz constant $\beta \gamma$.

Proof. Given $y \in \mathcal{M}$ we define a function $\varphi^{y}: \mathbb{N}_{0} \rightarrow \mathfrak{X}$ by $\varphi^{y}(k):=f\left(P^{k}(y)\right)$. Put $\varphi_{1}^{y}(k):=\varphi^{y}(k+1)$. It follows that $\varphi^{P(y)}(k)=f\left(P^{k}(P(y))\right)=f\left(P^{k+1}(y)\right)$ $=\varphi^{y}(k+1)=\varphi_{1}^{y}(k)$, which means

$$
\varphi^{P(y)}=\varphi_{1}^{y} \quad \text { for all } y \in \mathcal{M} .
$$

Now Theorem 1 implies that there is an invariant mean $M: \mathcal{B}\left(\mathbb{N}_{0}, \mathfrak{X}\right) \rightarrow \mathfrak{X}$. Define a function $F: \mathcal{M} \rightarrow \mathfrak{X}$ by the formula $F(x):=M\left(\varphi^{x}\right)$ for $x \in \mathcal{M}$. Hence, by (9) and (i) we get $F(P(x))=M\left(\varphi^{P(x)}\right)=M\left(\varphi_{1}^{x}\right)=M\left(\varphi^{x}\right)=F(x)$, so $F$ is $P$-periodic.

Finally, we show that $f$ is a Lipschitz function. Recall that the function $M$ is a continuous operator with $\|M\|=1$. From the definition of $F$, we have

$$
\begin{aligned}
\|F(x)-F(y)\|_{\mathfrak{X}} & =\left\|M\left(\varphi^{x}\right)-M\left(\varphi^{y}\right)\right\|_{\mathfrak{X}} \leqslant\|M\| \cdot\left\|\varphi^{x}-\varphi^{y}\right\|_{\infty} \\
& =\|M\| \cdot \sup \left\{\left\|\varphi^{x}(k)-\varphi^{y}(k)\right\|_{\mathfrak{X}}: k \in \mathbb{N}_{0}\right\} \\
& =\sup \left\{\left\|f\left(P^{k}(x)\right)-f\left(P^{k}(y)\right)\right\|_{\mathfrak{X}}: k \in \mathbb{N}_{0}\right\} \\
& \leqslant \sup \left\{\beta \varrho\left(P^{k}(x), P^{k}(y)\right): k \in \mathbb{N}_{0}\right\} \leq \beta \gamma \varrho(x, y) .
\end{aligned}
$$

The Proof of Theorem 5 is complete.

\section{Sandwich with functional equation $f \circ p=r \circ f$}

In the previous section we saw that the problem of determining periodic (resp. $p$-super-periodic) functions leads to the functional equation $f(p(x))=f(x)$ (resp. the functional inequality $f(p(x)) \geq f(x))$. So, it becomes quite natural to consider the functional equation $f(p(x))=r(f(x))$ (resp. the functional inequality $f(p(x)) \geq r(f(x)))$.

The results presented in this section are of the type as in the previous section, but focused on the functional equation $f(p(x))=r(f(x))$ instead of periodic functions. First we prove a sandwich type result for the functional equation $f(p(x))=g(f(x))$.

Theorem 6. Let $I \subseteq \mathbb{R}$ be a compact interval. Assume that functions $p: I \rightarrow I$ and $r: I \rightarrow I$ satisfy the following conditions: $p$ is invertible and

$$
\left|p^{-1}(x)-p^{-1}(y)\right| \leq \alpha(|x-y|), \quad x, y \in I,
$$

where $\alpha:[0, \infty) \rightarrow[0, \infty)$ is right-continuous at 0 and $\alpha(0)=0 ; r$ is increasing, continuous and

$$
|r(x)-r(y)| \leq \beta(|x-y|), \quad x, y \in I
$$


where $\beta:[0, \infty) \rightarrow[0, \infty)$ is increasing, right continuous at 0 and $\beta(0)=0$. Assume that there is a right-continuous at 0 function $\gamma:[0, \infty) \rightarrow[0, \infty)$, with $\gamma(0)=0$ such that

$$
\beta \circ \gamma \circ \alpha \leq \gamma
$$

If there exist continuous functions $f, g: I \rightarrow I$ such that

$$
\begin{gathered}
f \leq g, \\
f \circ p \leq r \circ f, \\
r \circ g \leq g \circ p,
\end{gathered}
$$

and for some $h_{0}: I \rightarrow I$, we have $f \leq h_{0} \leq g$ and $\left|h_{0}(x)-h_{0}(y)\right| \leq \gamma(|x-y|)$ for all $x, y \in I$, then there is a continuous function $h: I \rightarrow I$ such that

$$
f \leq h \leq g
$$

and satisfying the equality

$$
h \circ p=r \circ h .
$$

Proof. Let $\mathcal{C}(I)$ be the Banach space of all continuous functions $\varphi: I \rightarrow \mathbb{R}$ with the supremum norm $\|\varphi\|_{\infty}:=\sup \{|\varphi(x)|: x \in I\}$, and denote by $\mathcal{F}$ the family of all continuous functions $\varphi: I \rightarrow I$ such that

$$
f \leq \varphi \leq g,
$$

and

$$
|\varphi(x)-\varphi(y)| \leq \gamma(|x-y|), \quad x, y \in I .
$$

By the assumption, $\mathcal{F}$ is nonempty. Define the mapping $T: \mathcal{F} \rightarrow \mathcal{C}(I)$ by

$$
T(\varphi):=r \circ \varphi \circ p^{-1} .
$$

Then, for every $\varphi$ and $x \in I$, applying in turn: inequality (14) together with the invertibility of $p$; the monotonicity of $r$ and the inequality in (17); again the monotonicity of $r$ and the second inequality in (17); the inequality in (15), we obtain

$$
f(x) \leq r\left(f\left(p^{-1}(x)\right)\right) \leq r\left(\varphi\left(p^{-1}(x)\right)\right) \leq r\left(g\left(p^{-1}(x)\right)\right) \leq g(x),
$$

which shows that

$$
f \leq T(\varphi) \leq g
$$

Moreover, using in turn: the definition of $T$; inequality (11); inequality (10); and (12), we get for arbitrary $\varphi \in \mathcal{F}$ and for all $x, y \in I$,

$$
\begin{aligned}
|T(\varphi)(x)-T(\varphi)(y)| & =\left|r\left(\varphi\left(p^{-1}(x)\right)\right)-r\left(\varphi\left(p^{-1}(y)\right)\right)\right| \\
& \leq \beta\left(\left|\varphi\left(p^{-1}(x)\right)-\varphi\left(p^{-1}(y)\right)\right|\right) \\
& \leq \beta\left(\gamma\left(\left|p^{-1}(x)-p^{-1}(y)\right|\right)\right) \\
& \leq \beta(\gamma(\alpha(|x-y|))) \\
& \leq \gamma(|x-y|),
\end{aligned}
$$


which shows that, $T(\varphi)$ satisfies inequality (18) for every $\varphi \in \mathcal{F}$. This proves that $T$ maps $\mathcal{F}$ into itself. Clearly, $\mathcal{F}$ is a closed subset of $C(I)$. It follows from the continuity of $\gamma$ at 0 and $\gamma(0)=0$, and the Arzela Theorem that $\mathcal{F}$ is a compact subset of $C(I)$. Moreover, one should be able to verify with little effort that $\mathcal{F}$ is convex.

Now we show that $T$ is continuous. Take $\varphi_{n} \in \mathcal{F}, n \in \mathbb{N} ; \varphi \in \mathcal{F}$ such $\lim _{n \rightarrow \infty} \varphi_{n}=\varphi$. In turns making use the definition of the norm $\|\cdot\|_{\infty}$; the bijectivity of $p$; inequality (11); the monotonicity of $\beta$ and the continuity of the involved functions on the compact interval, we have

$$
\begin{aligned}
\left\|T\left(\varphi_{n}\right)-T(\varphi)\right\|_{\infty} & =\left\|r \circ \varphi_{n} \circ p^{-1}-r \circ \varphi \circ p^{-1}\right\|_{\infty} \\
& =\sup \left\{\left|r\left(\varphi_{n}\left(p^{-1}(x)\right)\right)-r\left(\varphi\left(p^{-1}(x)\right)\right)\right|: x \in I\right\} \\
& =\sup \left\{\left|r\left(\varphi_{n}(x)\right)-r(\varphi(x))\right|: x \in I\right\} \\
& \leq \sup \left\{\beta\left(\left|\varphi_{n}(x)-\varphi(x)\right|\right): x \in I\right\} \\
& \leq \beta\left(\max \left\{\left|\varphi_{n}(x)-\varphi(x)\right|: x \in I\right\}\right) \\
& =\beta\left(\left\|\varphi_{n}-\varphi\right\|_{\infty}\right),
\end{aligned}
$$

whence $\lim _{n \rightarrow \infty} T\left(\varphi_{n}\right)=T(\varphi)$, so $T$ is continuous. The Schauder Fixed-Point Theorem implies that there is a functional $h \in \mathcal{F}$ such that $T(h)=h$, i.e. $r \circ h \circ p^{-1}=h$. Thus $h \circ p=r \circ h$.

Next we would like to present an application of the above theorem to Hölder functions. For a number $q \in(0,1]$, by $\mathcal{H}_{q}(I)$ denote the space of Hölder continuous functions $\varphi: I \rightarrow \mathbb{R}$, that is such that

$$
|\varphi(x)-\varphi(y)| \leq C|x-y|^{q}, \quad x, y \in I,
$$

for some $C \geq 0$. As an immediate consequence of the above result we obtain:

Corollary 7. Let $I \subseteq \mathbb{R}, p, r, f, g, h_{0}$ satisfy the conditions of Theorem 6 with $\alpha(t)=\alpha(1) t, \beta(t)=\beta(1) t$ and $\gamma(t)=\gamma(1) t^{q}$, for some $q \in(0,1]$. If

$$
\beta(1) \leq[\alpha(1)]^{-q},
$$

then there exists a function $h \in \mathcal{H}_{q}(I)$ such that

$$
f \leq h \leq g \text {, }
$$

and satisfying the equality

$$
h \circ p=r \circ h .
$$

We conclude this work by proving two results concerning the functional equation $\alpha f(p(x))=g(f(x))$ and the functional inequality $\alpha f\left(p^{n}(x)\right) \geq g^{n}$ $(f(x))$.

Theorem 8. Let $\mathcal{M} \subseteq\left(\mathbb{R}^{n},\|\cdot\|\right)$ be a compact subset with int $\mathcal{M} \neq \emptyset$, and suppose that $\mathfrak{X}, \mathcal{W}, \mathcal{Z}$ are nontrivial Banach spaces such that $\mathfrak{X}=\mathcal{W}+\mathcal{Z}$ and $\mathcal{W} \cap \mathcal{Z}=\{0\}$, i.e. $\mathfrak{X}$ is the direct sum of $\mathcal{W}$ and $\mathcal{Z}$. Assume that $p: \mathcal{M} \rightarrow \mathcal{M}$ 
is continuous. Suppose that $g: \mathfrak{X} \rightarrow \mathfrak{X}$ is a continuous surjection such that the restriction $\left.g\right|_{\mathcal{W}}: \mathcal{W} \rightarrow \mathfrak{X}$ is a surjective isometry (not necessarily linear), and $\mathcal{Z} \subseteq\{x \in \mathfrak{X}: g(x)=0\}$. Then for every $\alpha \in(0,1)$ there exists a continuous function $f: \mathcal{M} \rightarrow \mathfrak{X}$ such that

$$
\forall_{x \in \mathcal{M}} \quad \alpha f(p(x))=g(f(x))
$$

and $f$ is not a constant function. Moreover, if $\operatorname{dim} \mathcal{Z} \geq n$, then the solution can be chosen in such a way that $f$ is injective.

Proof. Let $N: \mathbb{R}^{n} \rightarrow \mathcal{Z}$ be a continuous linear mapping with $\|N\| \leq 1$. The operator $N$ can be chosen in such a way that $\left.N\right|_{\mathcal{M}}$ is not constant. Since $N(\mathcal{M})$ is compact and $N(\mathcal{M}) \subseteq \mathcal{Z} \subseteq \mathfrak{X}$, without loss of generality, it may be assumed that the set $\mathcal{M}$ is contained in the closed unit ball $B_{\mathfrak{X}}:=\{y \in \mathfrak{X}:\|y\| \leq 1\}$. By $\mathcal{C}(\mathcal{M}, \mathfrak{X})$ we denote the space of continuous $\mathfrak{X}$-valued functions with the supremum norm $\|\cdot\|_{\infty}$, and set $B_{\mathcal{C}}:=\left\{f \in \mathcal{C}(\mathcal{M}, \mathfrak{X}):\|f\|_{\infty} \leq 1\right\}$.

Put $g_{1}:=\left(\left.g\right|_{\mathcal{W}}\right)^{-1}: \mathfrak{X} \rightarrow \mathcal{W}$ and define a mapping $\varphi: B_{\mathcal{C}} \rightarrow B_{\mathcal{C}}$ by the formula $\varphi(f):=\left.(1-\alpha) N\right|_{\mathcal{M}}+\alpha g_{1} \circ f \circ p$. It is easy to verify that $\varphi$ is a contraction. By the Banach Fixed-Point Theorem, there is a function $f_{\alpha}: \mathcal{M} \rightarrow$ $\mathfrak{X}$ such that $\left.(1-\alpha) N\right|_{\mathcal{M}}+\alpha g_{1} \circ f_{\alpha} \circ p=f_{\alpha}$.

Since $\left.g\right|_{\mathcal{W}}: \mathcal{W} \rightarrow \mathfrak{X}$ is a surjective isometry, obviously, so is $g_{1}: \mathfrak{X} \rightarrow \mathcal{W}$. The Mazur-Ulam Theorem implies that $g_{1}$ is an affine mapping. Since $g \circ g_{1}=\mathrm{Id}_{\mathfrak{X}}$, we have

$$
\begin{aligned}
g\left(f_{\alpha}(x)\right) & =g\left(\left.(1-\alpha) N\right|_{\mathcal{M}}(x)+\alpha\left(g_{1} \circ f_{\alpha} \circ p\right)(x)\right) \\
& =(1-\alpha) g\left(\left.N\right|_{\mathcal{M}}(x)\right)+\alpha g\left(g_{1}\left(f_{\alpha} \circ p(x)\right)\right) \\
& =(1-\alpha) \cdot 0+\alpha f_{\alpha}(p(x))=\alpha f_{\alpha}(p(x)),
\end{aligned}
$$

whence $g \circ f_{\alpha}=\alpha f_{\alpha} \circ p$. Now, we prove that $f_{\alpha}$ is not constant. Assume, contrary to our claim, that $f_{\alpha}(\cdot)=c$ for some $c \in \mathfrak{X}$. It follows that $\left(g_{1} \circ\right.$ $\left.f_{\alpha} \circ p\right)(\cdot)=g_{1}(c)$, whence $\left.(1-\alpha) N\right|_{\mathcal{M}}(\cdot)=c-\alpha g_{1}(c)$. Thus the function $\left.N\right|_{\mathcal{M}}: \mathcal{M} \rightarrow \mathcal{Z}$ would be constant, which is a contradiction.

Now assume that $\operatorname{dim} \mathcal{Z} \geq n$. Then there is a continuous injective linear operator $N: \mathbb{R}^{n} \rightarrow \mathcal{Z}$. Repeating the above reasoning we obtain the equality $\left.(1-\alpha) N\right|_{\mathcal{M}}+\alpha g_{1} \circ f_{\alpha} \circ p=f_{\alpha}$. We show that $f_{\alpha}$ is an injection. Suppose that $f_{\alpha}(x)=f_{\alpha}(y)$. Then

$$
(1-\alpha) N(x)+\alpha g_{1}\left(f_{\alpha} p(x)\right)=f_{\alpha}(x)=f_{\alpha}(y)=(1-\alpha) N(y)+\alpha g_{1}\left(f_{\alpha} p(y)\right) .
$$

Note that $\mathfrak{X}=\mathcal{W} \oplus \mathcal{Z}, g_{1}(\mathfrak{X}) \subseteq \mathcal{W}$ and $N(x), N(y) \in \mathcal{Z}$. So the uniqueness of the decomposition implies that $N(x)=N(y)$. Since $N$ is injective, $x=y$.

Bearing the Proof of Theorem 8 in mind, we can formulate and prove another result. More precisely, now we want to study the existence of solutions $f$ of the functional inequality $f(p(x)) \geq g(f(x))$. 
Theorem 9. Let $\mathcal{M} \subseteq\left(\mathbb{R}^{n},\|\cdot\|\right)$ be a compact convex subset. Assume that $p: \mathcal{M} \rightarrow \mathcal{M}$ is a continuous mapping. Let $0 \leq d<b<c<a$. Suppose that $g:[0, a] \rightarrow[0, a]$ is a continuous function such that $[d, b]=\{x \in[0, a]: g(x)=$ $0\}$ and assume that $\left.g\right|_{[0, c]}:[0, c] \rightarrow[0, a]$ is convex. Moreover, let $\left.g\right|_{[b, c]}:[b, c] \rightarrow$ $[0, a]$ be an increasing bijection and assume that $g_{1}:=\left(\left.g\right|_{[b, c]}\right)^{-1}$ is a Lipschitz function with Lipschitz constant $\operatorname{Lip}\left(g_{1}\right) \leq 1$. Then there exists a continuous function $f: \mathcal{M} \rightarrow \mathbb{R}$ such that

$$
\forall_{x \in \mathcal{M}} \forall_{n \in \mathbb{N}} g^{n}(f(x)) \leq \alpha f\left(p^{n}(x)\right) ;
$$

in particular $g \circ f \leq f \circ p$. Moreover, the solution $f$ can be chosen to be not constant. If $\mathcal{M} \subseteq \mathbb{R}$ is a compact interval and $p: \mathcal{M} \rightarrow \mathcal{M}$ is increasing, then $f$ can be chosen to be nondecreasing and not constant.

Proof. Fix a number $\alpha \in\left(\frac{b}{g_{1}(b)}, 1\right)$ and define a set

$$
W:=\{f: \mathcal{M} \rightarrow[b, a] \mid f \text { is continuous }\} .
$$

Consider a continuous mapping $\mu: \mathcal{M} \rightarrow[d, b]$. Suppose that $\mu$ is not constant. Define a mapping $\varphi: W \rightarrow W$ by $\varphi(f):=(1-\alpha) \mu+\alpha g_{1} \circ f \circ p$ for all $f \in W$.

The mapping $\varphi$ is well-defined. Indeed, since the range of of $f \circ p$ is bounded from below by $b$, the monotonicity of $g_{1}$ implies that $\varphi(f)(t)=(1-\alpha) \mu(t)+$ $\alpha g_{1}(f(p(t))) \geq \alpha g_{1}(b) \geq b$, so $\varphi(f)(t) \in[b, a]$ for every $t \in \mathcal{M}$. By the Banach Fixed-Point Theorem we get

$$
f=(1-\alpha) \mu+\alpha g_{1} \circ f \circ p
$$

for some $f \in W$. Since $g$ is convex and $g \circ \mu=0$, it follows from (19) that $g \circ f \leq \alpha f \circ p$. Combining this inequality and again (19), we get

$$
g \circ f \leq \alpha f \circ p \leq f \circ p=(1-\alpha) \mu \circ p+g_{1} \circ f \circ p^{2} .
$$

Since $g$ is increasing in $[b, c]$ and convex, we obtain $g \circ g \circ f \leq \alpha \circ f \circ p^{2}$, that $g^{2} \circ f \leq \alpha \circ f \circ p^{2}$. Now, taking into account that $g^{n}$ is increasing for $n \geq 2$, we can prove, by induction, that $g^{n} \circ f \leq \alpha \circ f \circ p^{n}$.

Moreover, if $\mathcal{M} \subseteq \mathbb{R}$ is a compact interval, then we may chose an increasing function $\mu: \mathcal{M} \rightarrow[d, b]$. Next we define

$$
W:=\{f: \mathcal{M} \rightarrow[b, a] \mid f \text { is continuous and nondecreasing }\} .
$$

and consider a similar mapping $\varphi: W \rightarrow W$. The rest run similarly. Finally, in this case it is easy to notice that the fixed point $f$ is a nondecreasing function. 
Open Access. This article is licensed under a Creative Commons Attribution 4.0 International License, which permits use, sharing, adaptation, distribution and reproduction in any medium or format, as long as you give appropriate credit to the original author(s) and the source, provide a link to the Creative Commons licence, and indicate if changes were made. The images or other third party material in this article are included in the article's Creative Commons licence, unless indicated otherwise in a credit line to the material. If material is not included in the article's Creative Commons licence and your intended use is not permitted by statutory regulation or exceeds the permitted use, you will need to obtain permission directly from the copyright holder. To view a copy of this licence, visit http:// creativecommons.org/licenses/by/4.0/.

Publisher's Note Springer Nature remains neutral with regard to jurisdictional claims in published maps and institutional affiliations.

\section{References}

[1] Ciepliński, K., Leśniak, Z.: On conjugacy equation in dimension one. In: Recent Developments in Functional Equation and Inequalities. Institute of Mathematics, vol. 99. Banach Center Publ., Polish Academy of Sciences, Warszawa, pp. 31-44 (2013)

[2] Ciepliński, K., Zdun, M.C.: On semi-conjugacy equation for homeomorphisms of the circle. In: Functional Equations-Results and Advances. Advanced Mathematical (Dordr.) 3, Kluwer Acad. Publ., Dordrecht, pp. 135-158 (2002)

[3] Ciepliński, K., Zdun, M.C.: On uniqueness of conjugacy of continuous and piecewise monotone functions. Fixed Point Theory Appl. Art. ID 230414 (2009)

[4] Gajda, Z.: Invariant means and representations of semigroups in the theory of functional equations, Prace Naukowe Uniwersytetu Ślasskiego, Katowice (1992)

[5] Izumi, H., Li, L., Matkowski, J., Wróbel, M.: Sandwich with periodicity. Aequ. Math. 93, 699-709 (2019)

[6] Kopeček, O.: On solvability of $f(p(x))=q(f(x))$ for given real functions $p, q$. Aequ. Math. 90, 471-494 (2016)

[7] Shi, Y.-G., Li, L., Leśniak, Z.: On conjugacy of r-modal interval maps with nonmonotonicity height equal to 1. J. Differ. Equ. Appl. 19, 573-584 (2013)

[8] Zdun, M.C.: On conjugacy of multivalent functions on the circle. Rocznik Nauk.Dydakt. Prace Mat 17, 271-282 (2000)

[9] Zdun, M.C.: On conjugacy of some systems of functions. Aequ. Math. 61, 239-254 (2001)

[10] Zdun, M.C.: On conjugacy of homeomorphisms of the circle possessing periodic points. J. Math. Anal. Appl. 330, 51-65 (2007)

Janusz Matkowski

Faculty of Mathematics, Computer Science and Econometrics

University of Zielona Góra

Szafrana 4A

65-516 Zielona Góra

Poland

e-mail: J.Matkowski@wmie.uz.zgora.pl 
Paweł Wójcik

Institute of Mathematics

Pedagogical University of Cracow

Podchorążych 2

30-084 Kraków

Poland

e-mail: pawel.wojcik@up.krakow.pl

Received: June 18, 2019

Revised: January 20, 2020 